\title{
Criteria for return to running after anterior cruciate ligament reconstruction: a scoping review
}

\author{
Alexandre J M Rambaudd, ${ }^{1,2}$ Clare L Ardern, ${ }^{3,4}$ Patricia Thoreux, ${ }^{5,6}$ \\ Jean-Philippe Regnaux, ${ }^{7,8}$ Pascal Edouard ${ }^{1,9}$
}

- Additional material is published online only. To view please visit the journal online (http://dx.doi.org/10.1136/ bjsports-2017-098602).

${ }^{1}$ Inter-University Laboratory of Human Movement Biology (LIBM EA 7424), University of Lyon, University Jean Monnet, Saint Etienne, France ${ }^{2}$ Physiotherapy Clinic of the Sport Center, La Talaudière, France

${ }^{3}$ Division of Physiotherapy, Linköping University, Linköping, Sweden

${ }^{4}$ School of Allied Health, La Trobe University, Melbourne, Victoria, Australia

${ }^{5}$ Orthopaedic Department and Sport Medicine Unit, APHPUniversity Paris, Bobigny, France ${ }^{6}$ Institut de Biomécanique Humaine Georges Charpak, Arts et Métiers ParisTech, Paris, France

${ }^{7}$ French School of Public Health (EHESP), Paris, France ${ }^{8}$ Inserm U1153, Centre de Recherche Epidémiologie et Statistique Sorbonne Paris Cité (CRESS), Paris, France ${ }^{9}$ Sports Medicine Unit, Department of Clinical and Exercise Physiology, Faculty of Medicine, University Hospital of Saint-Etienne, Saint-Etienne, France

\section{Correspondence to}

Alexandre J M Rambaud, InterUniversity Laboratory of Human Movement Biology (LIBM EA

7424), University of Lyon, SaintPriest en Jarez 42270, France; alexandre.rambaud.kine@gmail. com

Accepted 30 March 2018 Published Online First 2 May 2018

Check for updates

To cite: Rambaud AJM,

Ardern $\mathrm{CL}$, Thoreux $\mathrm{P}$

et al. Br J Sports Med

2018:52:1437-1444.

\section{ABSTRACT}

Objective To describe the criteria used to guide clinical decision-making regarding when a patient is ready to return to running (RTR) after $A C L$ reconstruction.

Design Scoping review.

Data sources The MEDLINE (PubMed), EMBASE, Web of Science, PEDro, SPORTDiscus and Cochrane Library electronic databases. We also screened the reference lists of included studies and conducted forward citation tracking.

Eligibility criteria for selecting studies Reported at least one criterion for permitting adult patients with primary $A C L$ reconstruction to commence running postoperatively.

Results 201 studies fulfilled the inclusion criteria and reported 205 time-based criteria for RTR. The median time from when RTR was permitted was 12 postoperative weeks (IQR=3.3, range 5-39 weeks). Fewer than one in five studies used additional clinical, strength or performance-based criteria for decision-making regarding RTR. Aside from time, the most frequently reported criteria for RTR were: full knee range of motion or $>95 \%$ of the non-injured knee plus no pain or pain $<2$ on visual analogue scale; isometric extensor limb symmetry index (LSI)>70\% plus extensor and flexor LSI>70\%; and hop test $\mathrm{LSI}>70 \%$.

Conclusions Fewer than one in five studies reported clinical, strength or performance-based criteria for RTR even though best evidence recommends performance-based criteria combined with time-based criteria to commence running activities following $\mathrm{ACL}$ reconstruction.

\section{INTRODUCTION}

After ACL reconstruction, ${ }^{1-3}$ an important milestone is returning to running (RTR). The RTR is a key to the participation element of the return to sport continuum (figure 1 ). ${ }^{2}$ Running places relatively low demands on the knee. ${ }^{4}$ However, RTR marks the beginning of the transition from impairment-focused tasks in early rehabilitation (eg, knee range of motion (RoM) exercises, isometric quadriceps strengthening) to the functional, sport-specific tasks that characterise more advanced rehabilitation (eg, sprinting, pivoting, cutting). ${ }^{56}$

There is a lack of information regarding when the patient can RTR following ACL reconstruction. Steady progress through high-quality rehabilitation influences functional outcomes, ${ }^{7}$ and premature return to sport increases the risk for reinjury. ${ }^{8}$ Conversely, delayed progress may hinder motivation and psychological readiness to return to sport. ${ }^{9}$
Therefore, the aims of this scoping review were: (1) to describe the criteria used in clinical decision-making for RTR after ACL reconstruction; (2) to report how these criteria have changed over time alongside changes in surgical and rehabilitation approaches; and (3) to provide information to help clinicians and patients make quality decisions regarding returning to running after ACL reconstruction.

\section{METHODS}

We conducted a scoping review because this approach is superior to a systematic review for addressing an exploratory research question. ${ }^{10} 11$ Systematic reviews aim to facilitate synthesis of a known field of research to answer questions regarding the effectiveness of a specific intervention for a particular problem. In contrast, scoping reviews map key concepts, types of evidence and gaps in the research in a particular field. As for a systematic review, a scoping review involves systematically searching, selecting and synthesising research evidence. ${ }^{11}$ We followed the methodological framework proposed by Arksey and O'Malley ${ }^{10}$ and the Joanna Briggs Institute $^{12}$ when conducting and reporting this scoping review.

Our research question was: 'Which criteria are used to determine when the patient can return to running following primary ACL reconstruction?'

We limited our scoping review to primary ACL reconstruction because the outcomes of revision surgery are typically inferior to the outcomes of primary surgery. ${ }^{13} 14$

\section{Selection criteria}

To select articles for inclusion in this scoping review, the following criteria were employed:

Type of studies

We included: sources of information as recommended in the manual 'Methodology for JBI Scoping Reviews' ${ }^{12}$ (including primary research studies, reviews, systematic reviews, scoping reviews, meta-analyses, guidelines, rehabilitation protocol, cases series, clinical commentaries and technical notes) dealing with human subjects, with a rehabilitation programme reported or including criteria for progression to running or jogging.

Only studies published in English language were included. This restriction was based on findings from systematic reviews suggesting no evidence of bias for conventional medicine if studies written in languages other than English were excluded. ${ }^{15} 16$ 


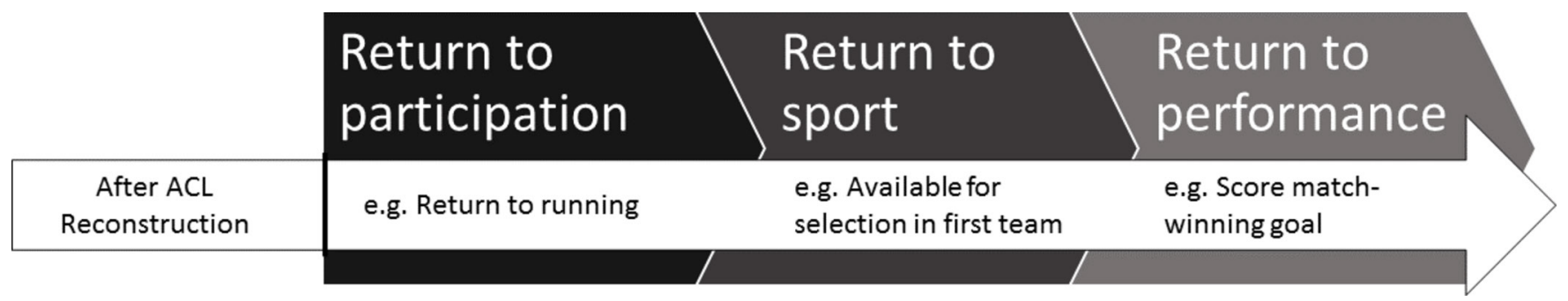

Figure 1 Examples of the three elements of the return to sport (RTS) continuum from Ardern et al ${ }^{2}$ in the specific context of ACL reconstruction.

We excluded: conference abstracts, opinion pieces, magazine and newspaper articles.

\section{Types of participants}

We included: studies of skeletally mature patients or people performing physically demanding work with primary ACL reconstruction (autograft, with or without meniscus surgery).

We excluded: studies of patients with knee dislocation, patients undergoing revision ACL reconstruction and studies including only patients who were more than 40 years old at the index ACL reconstruction (middle-aged patients with joint trauma may be more likely to have knee osteoarthritis, ${ }^{17} 18$ and this may influence the contents and progression of rehabilitation).

\section{Types of interventions}

We excluded: studies of patients undergoing ACL reconstruction plus major concomitant procedures (eg, articular cartilage surgery, posterior cruciate ligament surgery, lateral or medial collateral ligament surgery). One reason for choosing an allograft is the reduced donor site morbidity compared with autograft harvest. ${ }^{19}$ Reduced donor site morbidity may mean that rehabilitation milestones may be different (accelerated) compared with milestones for patients who choose an autograft. Therefore, it is possible that the time criterion for RTR might be different for patients who had an allograft. Excluding studies in which allografts were the sole graft option reduced the potential clinical heterogeneity in the included studies. In addition, since most patients have ACL reconstruction using an autograft, ${ }^{20} 21$ excluding allografts reduced the clinical heterogeneity in the results of our scoping review.

\section{Types of outcomes}

We included: clinical outcomes or description of RTR criteria: criteria defined by timeline, questionnaires, impairment assessments, isometric or isokinetic strength assessment, or performance-based assessments.

\section{Study selection}

Our search strategy involved a three-step approach ${ }^{22}$ :

\section{Step 1: initial limited search}

We conducted a pilot search (in October 2016) in the MEDLINE and SPORTDiscus electronic databases using the terms 'anterior cruciate ligament' AND 'reconstruction' AND 'run' (online supplementary file A).

\section{Step 2: identify keywords and index terms}

We analysed the title, abstract and index terms used to describe the studies identified in step 1 to identify appropriate keywords for inclusion in the final search strategy (online supplementary file B).
Step 3: execution of final search strategy and further searching of references and citations

In November 2016, we searched the MEDLINE (PubMed), EMBASE, Web of Science, PEDro, SPORTDiscus and The Cochrane Library electronic databases using the search strategy we had developed. We also screened the reference lists of included studies, the reference lists of key systematic reviews and narrative reviews, and conducted forward citation tracking using Google Scholar in order to identify any potentially relevant studies that may have been missed in the electronic database search.

All articles were downloaded to the Covidence reference management platform (Covidence, http:/www.covidence. org), cross-referenced and any duplicates deleted before the selection criteria were applied. Two independent reviewers (AJMR and PE) first screened articles for eligibility based on the title and abstract (figure 1). Then, the full text was screened for inclusion in the scoping review by the same reviewers independently. Any discrepancies were resolved during a consensus meeting. A third reviewer was available if needed, but was not required.

\section{Data extraction}

Two reviewers (AJMR and PE) independently extracted data based on the following eight categories:

A. Authors and year of publication.

B. Origin (country in which the study was conducted).

C. Aim(s) of the study.

D. Study population (age, sex) and sample size (if applicable).

E. ACL reconstruction surgical technique (arthroscopy or open surgery, graft type).

F. Rehabilitation protocol: postoperative immobilisation (protective) or no postoperative immobilisation (contemporary).

G. Time-based criterion (weeks) from when patients were permitted to commence running activity (jogging or running on a treadmill, jogging or running on the field).

H. Other criteria: questionnaire-based or assessment-based criterion or criteria to allow the patient to commence running or jogging (eg, clinical assessment, isometric strength assessment, isokinetic strength assessment, functional goal-based assessment (performance-based assessment)).

To define if a rehabilitation protocol was protective or contemporary, ${ }^{23}$ we made a judgement based on the early rehabilitation phase: Protective rehabilitation protocols were characterised by a period of postoperative time where knee RoM was controlled (eg, with a cast), non-weightbearing or only toe-touch weightbearing and avoidance of active quadriceps in terminal extension. ${ }^{24} 25$ Contemporary rehabilitation protocols were characterised by no restrictions on mobilisation, ${ }^{25-27}$ encouragement of full active knee extension RoM, quadriceps activation and early weightbearing. 


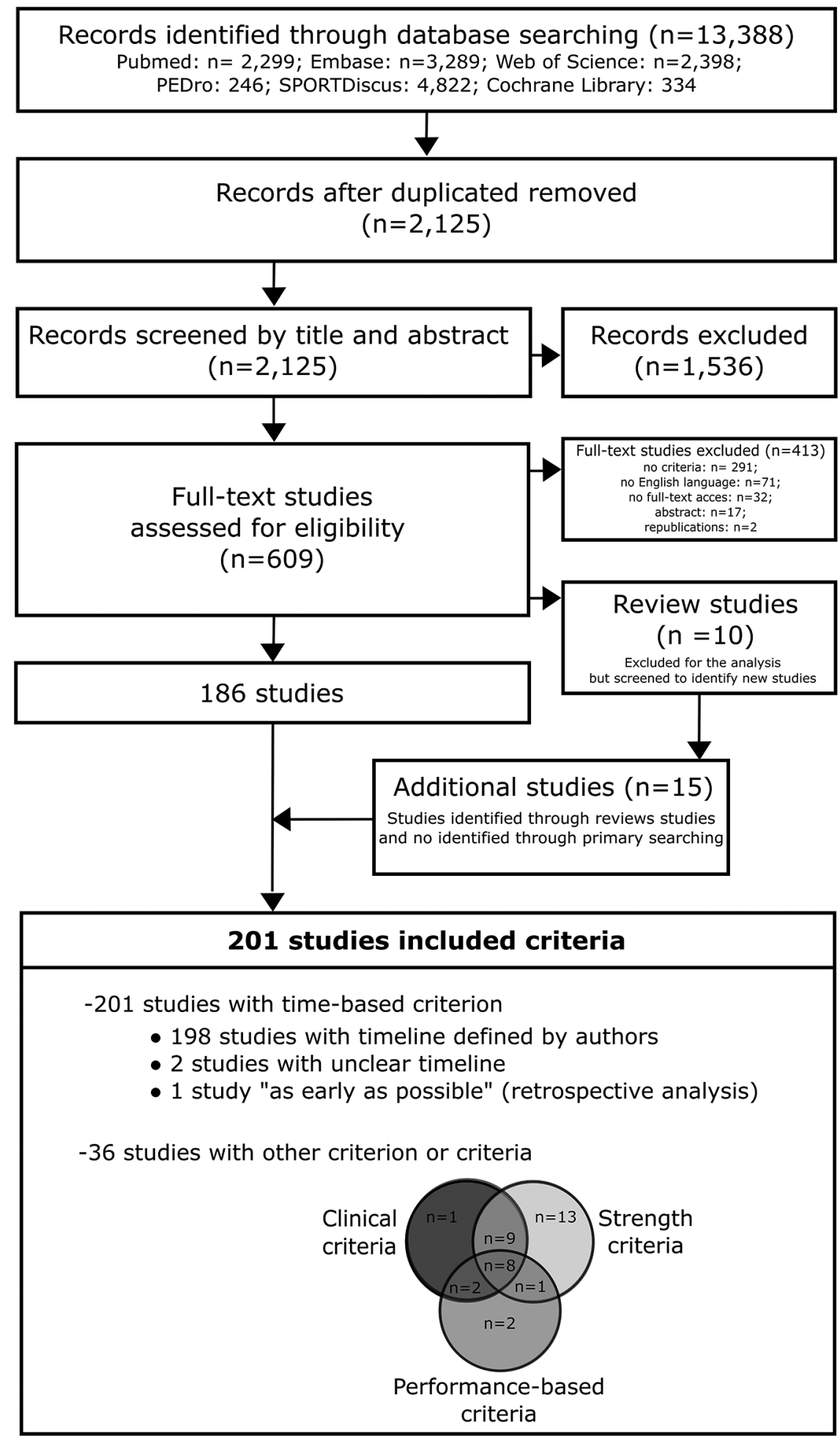

Figure 2 Flow chart of the study selection process.

\section{Assessment of risk of bias}

Since we made a descriptive analysis of variables representing choice and habits of the authors of included studies and not the results of an intervention administered to a population, we contend that our descriptive summary is unlikely to be adversely influenced by the internal validity of included studies. Therefore, we did not evaluate the internal validity of included studies.

\section{Data management}

Data were summarised and tabulated according to the eight data extraction categories (online supplementary file C). Qualitative variables were described by number (n) and percentage (\%). As there is no consensus in the terms used to describe RTR, and given the variety of terms found in the pilot search, time-based criteria were described by median and IQR to ensure that results were not influenced by outliers. 

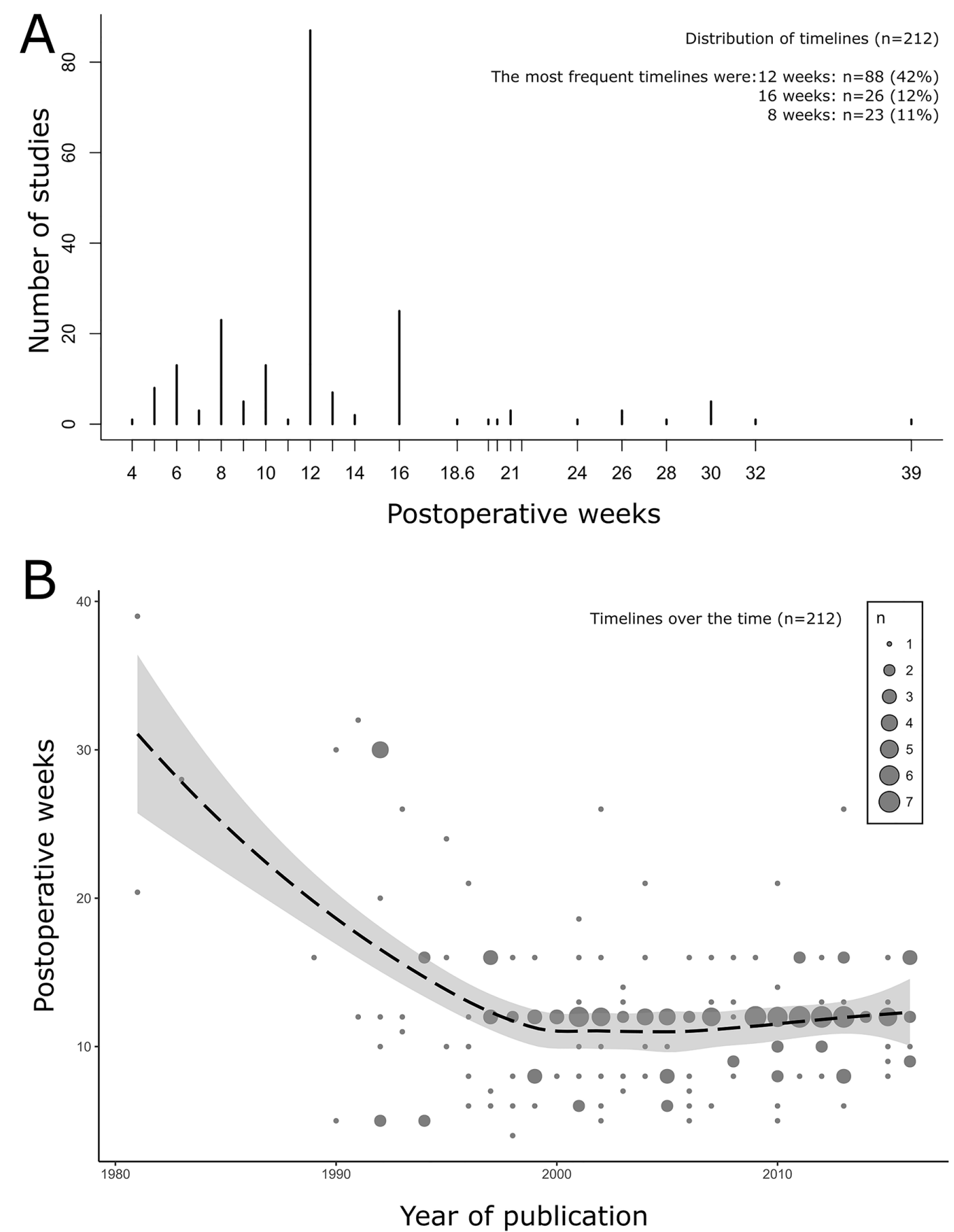

Figure 3 Distribution of time to permit return to running (RTR) (A) and timelines over the years $(B)(n=212)$ from studies included in the scoping review ( $n=198)$, with non-linear regression and $\mathrm{Cl}(95 \%)$ representing the time-based criteria trend of the authors during recent years.

If a study compared two protocols with different time-based criteria or other criteria, all criteria were included in the summary. If a study reported the time to RTR, the median or the average was included in the analysis. We also assessed if there was a change over time in the time-based criterion by using a non-linear Local Regression with 95\% CI (span $=0.75$, two degrees of polynomials). To be as exhaustive as possible, we retained open and arthroscopic surgical procedures and all rehabilitation approaches (dichotomised as protective or contemporary rehabilitation). Medians and IQR were calculated for the four groups (open surgery, arthroscopic surgery, protective rehabilitation and contemporary rehabilitation). Analyses were performed using the software R (http://www. R-project.org).

\section{RESULTS}

The flow chart of study selection is detailed in figure 2 (lists of excluded articles are presented in online supplementary files D and E). We selected and included 201 studies in our scoping review (figure 2).

\section{Time-based criteria used to clear the patient to RTR following $\mathrm{ACL}$ reconstruction}

Among the 201 studies reporting information on time-based criteria, 198 (99\%) reported in the methods or in the rehabilitation programme a time from which RTR was permitted, 2 studies (1\%) were unclear ${ }^{528}$ and 1 study made a retrospective analysis of the timeline. ${ }^{29}$ Since 13 studies included two groups 


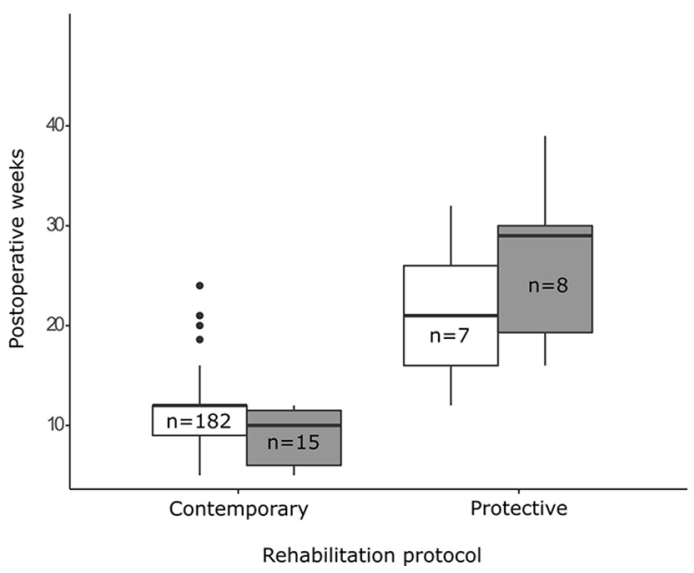

Figure 4 Box plot of the distribution of timeline of the two types of surgery (open surgery represented by grey boxes, arthroscopic surgery represented by white boxes) with the type of protocol of rehabilitation, including the number ( $\mathrm{n}$ ) with reported timelines for each group.

with a different timeline, a total of 212 timelines were included in the statistical analysis.

The median time from which RTR was permitted was 12 postoperative weeks ( $\mathrm{IQR}=3.3$, range 5-39 weeks) (figure 3A). The criteria used over time are presented in figure $3 \mathrm{~B}$ with the non-linear regression curve and the 95\% CI.

In the subgroup 'open surgery plus protective rehabilitation', the median time for RTR was 29 weeks ( $\mathrm{IQR}=10.7$ weeks). For the 'arthroscopic surgery plus protected rehabilitation' subgroup, the median time for RTR was 21.0 weeks $(\mathrm{IQR}=10.0$ weeks). There was no difference in the median time for RTR between open surgery and arthroscopic surgery when a contemporary rehabilitation protocol was employed (open surgery plus contemporary rehabilitation: 10 weeks, $\mathrm{IQR}=3.3$ weeks; arthroscopic surgery plus contemporary rehabilitation: 12 weeks, IQR=3.0 weeks) (figure 4).

\section{Assessment-based criteria for decision-making for RTR following $\mathrm{ACL}$ reconstruction}

Thirty-six studies (18\% of 201) reported using specific criteria to clear patients to RTR after ACL reconstruction. Sixteen studies ( $8 \%$ of 201 ) reported using a single criterion (clinical, strength or performance-based criterion), 12 studies (6\% of 201) reported using two types of criteria and eight studies (4\% of 201) reported using all three types of criteria (figure 2).

\section{Clinical criteria and questionnaires}

Twenty studies (10\% of 201$)$ reported using clinical criteria (table 1). The most common criteria were: knee flexion RoM $(n=14)$, knee effusion $(n=10)$ and pain $(n=10)$. The most quantifiable and reproducible criteria used were full knee RoM or RoM greater than $95 \%$ of the non-injured knee $(n=10)$, and pain $<2$ on visual analogue scale $(n=9)$.

\section{Strength criteria}

Thirty studies (15\% of 201) reported using strength criteria. Eleven used an isometric test criterion, 11 used an isokinetic test criterion and two used isometric and isokinetic test criteria (table 2). ${ }^{2730}$ The most common objective criteria were isometric quadriceps limb symmetry index (LSI; calculated by dividing the operated limb score by the non-operated limb score) $>80 \%$ $(\mathrm{n}=3)$ and isokinetic quadriceps and hamstrings LSI $>70 \%$ $(n=4)$ (table 2).

\begin{tabular}{|c|c|}
\hline Questionnaire score (n (\%)) & $2(1 \%)$ \\
\hline Self-report knee scoring & 2 \\
\hline Modified Noyes system $\geq 90^{46}$ & 1 \\
\hline $\begin{array}{l}\text { International Knee Documentation Committee subjective knee } \\
\text { form }>70^{5}\end{array}$ & 1 \\
\hline Clinical criteria (n (\%)) & $20(10 \%)$ \\
\hline Knee flexion range of motion (RoM) & $14(7 \%)$ \\
\hline RoM $>95 \%$ of the other side or full range of motion $2838-4046-5061$ & $10(5 \%)$ \\
\hline RoM $>120^{\circ 24} 41$ & $2(1 \%)$ \\
\hline RoM $>100^{\circ 4243}$ & $2(1 \%)$ \\
\hline Effusion & $10(5 \%)$ \\
\hline No effusion ${ }^{242843-4549}$ & $6(3 \%)$ \\
\hline Effusion of trace or less ${ }^{639-41}$ & $4(2 \%)$ \\
\hline Pain & $10(5 \%)$ \\
\hline No pain or $<2 / 10$ on visual analogue scale $e^{242838-45}$ & $10(5 \%)$ \\
\hline Knee extension RoM & $6(3 \%)$ \\
\hline Full RoM $39-424750$ & $6(3 \%)$ \\
\hline Clinical examination (others) & $5(3 \%)$ \\
\hline Satisfactory clinical examination 465962 & $3(1 \%)$ \\
\hline '... no inflammation... ${ }^{61}$ & $1(0.5 \%)$ \\
\hline No evidence of patellofemoral symptoms ${ }^{28}$ & $1(0.5 \%)$ \\
\hline Graft evaluation & $4(2 \%)$ \\
\hline Negative pivot shift and no postsurgical history of giving way 2845 & $2(1 \%)$ \\
\hline Anterior translation $<0.5 \mathrm{~mm}^{6259}$ & $2(1 \%)$ \\
\hline Ambulation & $2(1 \%)$ \\
\hline Full weightbearing ambulation ${ }^{41} 42$ & $2(1 \%)$ \\
\hline
\end{tabular}

\section{Performance-based criteria}

Thirteen studies (6\% of 201) reported using performance-based criteria: balance criteria $(n=6)$, normal gait pattern during walking or jog-in-place $(n=5)$, or functional tests $(n=7)$ (table 3). The objective criteria used were proprioception LSI of $100 \%$, composite score on Y-balance test $>90 \%$, functional test $\mathrm{LSI}>70 \%$, hop test LSI $>85 \%$, and two combined tests as 10 consecutive single-leg squats to $45^{\circ}$ knee flexion without loss of balance, and 30 step-up-and-holds without loss of balance or excessive motion outside of the sagittal plane (table 3 ).

\section{DISCUSSION}

In this scoping review that included 201 articles, time was the most frequently reported criterion for clearing patients to RTR after primary ACL reconstruction. The median time for clearance to RTR was 12 postoperative weeks ( $\mathrm{IQR}=3.3$, range 5-39 weeks). The time criterion for RTR has changed over the years mirroring changes in rehabilitation approaches. Fewer than one in five studies reported clinical, strength or performance-based criteria for RTR, the most frequently reported being: full knee RoM or $>95 \%$ of the non-injured knee; pain $<2$ on visual analogue scale; isometric lower limb strength LSI $>70 \%$; isokinetic quadriceps and hamstring strength LSI $>70 \%$; and hop test LSI $>70 \%$.

\section{Time-based criteria for RTR must respect biological healing of the ACL graft}

All included studies used at least a time-based criterion for RTR after ACL reconstruction. The median was 12 weeks $(\mathrm{IQR}=3.25)$, although there were three arbitrary time points reported in 65\% of included studies (figure 3A): 8 weeks (11\% 


\begin{tabular}{|c|c|}
\hline Strength criteria (n (\%)) & $30(15 \%)$ \\
\hline Isometric test criteria (studies, n) & $13(6 \%)$ \\
\hline$L S \mid>60 \%{ }^{3940}$ & $2(1 \%)$ \\
\hline $\mathrm{LSI}>65 \%^{30}$ & 1 \\
\hline LSI $>70 \%{ }^{262854}$ ('isometric leg press testing') $)^{70}$ & $4(3 \%)$ \\
\hline $\mathrm{LSI}>85 \%{ }^{43}$ & $1(0.5 \%)$ \\
\hline Quadriceps LSI>60\% ${ }^{40}$ & $1(0.5 \%)$ \\
\hline Quadriceps LSI>70\% ${ }^{42}$ & $1(0.5 \%)$ \\
\hline Quadriceps $L S \mid>75 \%^{33}$ & $1(0.5 \%)$ \\
\hline Quadriceps LSI>80\% ${ }^{64750}$ & $3(2 \%)$ \\
\hline Isotonic test criteria (studies, n) & $2(1 \%)$ \\
\hline LSI $>50 \%$ (with 'Push strength on the leg press', $n=1)^{39}$ & 1 \\
\hline LSI $>80 \%$ ('1-repetition maximum on the leg press $\left.\left(90-0^{\circ}\right)^{\prime}, n=1\right)^{34}$ & 1 \\
\hline Isokinetic test criteria (studies, n) & $13(6 \%)$ \\
\hline Hamstrings:quadriceps ratio between $60 \%$ and $70 \%{ }^{71}$ & 1 \\
\hline $\begin{array}{l}\text { Quadriceps } \mathrm{LSI}>80 \% \text {, hamstrings } \mathrm{LSI}>110 \% \text {, quadriceps torque/ } \\
\text { BW ratio }>55 \% \text {, hamstrings:quadriceps ratio }>70 \%{ }^{46}\end{array}$ & 1 \\
\hline Quadriceps and hamstrings $L S I>65 \%^{304572}$ & $3(2 \%)$ \\
\hline Quadriceps and hamstring $L S I>70 \%{ }^{25} 555670$ & $4(2 \%)$ \\
\hline Quadriceps and hamstring $L S I>75 \%^{24}$ & 1 \\
\hline $\begin{array}{l}\text { Quadriceps torque/BW ratio at } 300 \% / \mathrm{s:}(>40 \% \text { (men) and }>30 \% \\
\text { (women)) and quadriceps torque/BW ratio at } 180 \% / \mathrm{s}:(>60 \% \text { (men) } \\
\text { and }>50 \% \text { (women) })^{5}\end{array}$ & 1 \\
\hline$'$... satisfactory isokinetic test... ${ }^{15962}$ & $2(1 \%)$ \\
\hline Other subjective or unclear criteria (studies, n) & $6(3 \%)$ \\
\hline \multicolumn{2}{|l|}{ 'Good manual strength test'38 49} \\
\hline '...Lift 15 lbs during short arc quadriceps exercise... ${ }^{71}$ & 1 \\
\hline $\begin{array}{l}\text { 'Good muscle strength and control' (...when muscle strength and } \\
\text { control allow....) }\end{array}$ & 1 \\
\hline '...Sufficient musculature... ${ }^{.74}$ & 1 \\
\hline '...Good quadriceps contraction in extension....'41 & 1 \\
\hline
\end{tabular}

BW, body weight; LSI, limb symmetry index.

of included studies), 12 weeks (42\% of included studies) and 16 weeks ( $12 \%$ of included studies).

These three arbitrary time points might reflect different rehabilitation goals. Early RTR (ie, around 8 postoperative weeks) might reflect a focus on early return to sport. In contrast, late

\begin{tabular}{|c|c|}
\hline Performance-based criteria ( $(\%))$ & $13(7 \%)$ \\
\hline Balance (studies, n) & $6(3 \%)$ \\
\hline $\begin{array}{l}\text { One balance exercise with good control without objective criteria }{ }^{41} \\
435960\end{array}$ & $4(2 \%)$ \\
\hline Proprioception test $L S I=100 \%{ }^{46}$ & 1 \\
\hline Composite score on Y-balance test $\geq 90 \%{ }^{34}$ & 1 \\
\hline Gait pattern (studies, n) & $5(3 \%)$ \\
\hline Normal gait pattern 2834444750 & $5(3 \%)$ \\
\hline Functional test (studies, n) & $7(4 \%)$ \\
\hline Functional test $\mathrm{LSI}>70 \%{ }^{5962}$ & $2(1 \%)$ \\
\hline Hop tests $L S I \geq 85 \%{ }^{46}$ & 1 \\
\hline Hop tests or vertical jump tests without criteria ${ }^{61}$ & 1 \\
\hline Jog on a minitrampoline for $10 \mathrm{~min}$ without problems ${ }^{63}$ & 1 \\
\hline $\begin{array}{l}10 \text { consecutive single-leg squats to } 45^{\circ} \text { knee flexion without loss } \\
\text { of balance, and } 30 \text { steps-and-holds without loss of balance or } \\
\text { excessive motion outside of the sagittal plane }{ }^{34}\end{array}$ & 1 \\
\hline $\begin{array}{l}\text { Ability to perform single-limb functional exercises without pain or } \\
\text { swelling }\end{array}$ & 1 \\
\hline
\end{tabular}

RTR (ie, around 16 postoperative weeks) might reflect a focus on protecting the healing ACL graft.

While our understanding of graft healing timelines in humans is limited, ${ }^{31}$ the ACL graft may undergo substantial change in mechanical properties during the period from 8 to 16 postoperative weeks. ${ }^{3132}$ There is also wide variation in strength and functional recovery during this time. ${ }^{33-35}$ Therefore, there are likely different risks to the ACL graft and different capacities among patients to resume running. Collectively, these facts underscore the need for high-quality individualised rehabilitation and decision-making regarding rehabilitation progression-there is no one-size-fits-all recipe. ${ }^{36}$

\section{Clinical criteria are important indicators of adequate loading when returning to running}

Fewer than one in every five studies included in this scoping review reported information regarding assessment-based criteria (ie, clinical tests, strength tests, performance tests) used to guide decision-making regarding RTR. This might suggest that many authors consider time a sufficient sole criterion for RTR. On the other hand, it might also suggest there are few reliable and valid criteria for RTR. Only one in every 10 studies reported clinical criteria-the most frequently reported were knee RoM, effusion and pain. Current evidence-based rehabilitation guidelines for ACL reconstruction ${ }^{37}$ recommend patients have no effusion, active knee extension RoM of at least $0^{\circ}$ and normalised gait pattern to progress from early impairment-based rehabilitation to the second phase (heavy strength training, neuromuscular training and sport-specific training). It is possible that abnormal clinical examination was considered a contraindication to commence running (and simply not reported in the published article), and this might explain why there was a low proportion of studies citing clinical criteria for RTR.

We suggest that these clinical criteria: pain $<2$ on visual analogue scale, ${ }^{24} 28$ 38-45 $95 \%$ knee flexion RoM, ${ }^{28}{ }^{38-40} 46-51$ full extension RoM $\left(0^{\circ}\right.$ knee extension), ${ }^{39-424750}$ no effusion/trace of effusion, ${ }^{6242839-4143-4549}$ should be used as 'non-negotiable' clinical milestones for RTR-patients must fulfil these criteria before RTR as a way to ensure adequate loading. The knee must have sufficient capacity, first to cope with the demands of activities of daily living and rehabilitation, before commencing higher level functional tasks including running. Therefore, a symptomatic knee (that may be characterised by an increase in pain, effusion and RoM restrictions) after walking, strengthening and/or sensorimotor control exercises suggests inferior load management. In this case, delaying RTR is appropriate, irrespective of the postoperative time.

\section{Strength and performance-based criteria are relevant parameters to judge the patient's capacity to RTR}

Strength was the most frequently reported category of RTR criteria $(n=31,16 \%)$. Since the hamstring and quadriceps have an important role in the active stabilisation of the knee ${ }^{52}$ and in motor control strategy, ${ }^{53}$ it seems reasonable to include strength criteria in RTR decision-making. Adequate quadriceps strength and hamstrings strength are also important criteria for safe return to sport decision-making. ${ }^{51}{ }^{52}$ The most frequent recommendations for the strength evaluation for RTR were: (1) isometric assessment: an LSI of lower limb $>70 \%{ }^{26-28} 54$ and quadriceps LSI $>80 \%{ }^{6344750}$; (2) for isokinetic assessment: quadriceps and hamstrings LSI $>70 \% .25275556$

Only 17 articles (7\%) used performance-based criteria for clearance to RTR, despite the fact that running represents a 
functional progression from the impairments that early rehabilitation focuses on addressing. ${ }^{25758}$ Equally, despite the fact that adequate postural control and normal gait pattern are important precursors for RTR, ${ }^{283441434447505960}$ there was no assessment of gait reported in the included articles. For example, walking with a gait deviation might suggest inferior load management, and alert the clinician that the patient may not be ready to RTR.

Functional tests replicating some of the physical requirements for running may include hop tests performed with a controlled and balanced landing. ${ }^{46596162}$ Other functional tests, such as single-limb squats at $45^{\circ}$ knee flexion, performed without loss of balance and without dynamic knee valgus, or step-up-andholds, ${ }^{34}$ may also be appropriate tests for determining whether the patient is ready to RTR. These tests, described with precise instructions about the quality of the knee flexion (without knee valgus), are simple tests, require little equipment and have the advantage of doubling as rehabilitation exercises. Balance tests that aim to assess aspects of movement quality (eg, Y-balance test and modified Star Excursion Balance Test) ${ }^{34}$ may also provide information regarding movement control that is relevant for RTR, and complements the other tests mentioned previously.

Quality of movement performance is equally as important as quantity, and performance-based tests allow the clinician to assess both aspects. This information is useful for decision-making regarding RTR, and for further progressions during rehabilitation (eg, commencing sport-specific training). Therefore, it seems reasonable to suggest that quality decision-making regarding functional progressions in rehabilitation (including RTR) should be individualised and based on a test battery comprising multiple criteria including function, strength and time. ${ }^{37}$ Frequent functional testing, using a range of different tests, that starts early in the rehabilitation process, characterises high-quality rehabilitation. ${ }^{36}$ This information provides important feedback to the clinician that can be subsequently used to target specific deficits and make changes, as necessary, in a timely way. Clinicians are not clairvoyants-without regular assessment, it is impossible to know whether the planned rehabilitation is high quality and appropriate for the individual patient.

We suggest that the clinician consider for strength and performance-based criteria: hamstring LSI and quadriceps LSI $>70 \%$ evaluated by isometric assessments, and hop test LSI $>70 \%$. The addition of a single-leg squat or step-up assessment performed without increase in knee valgus may also be considered. Further research is needed to help guide the clinician regarding which combination of strength and performance-based criteria should be used to assess whether the patient is ready to RTR.

\section{Brace or no brace to RTR?}

Only six of the 201 included studies ${ }^{63-68}$ compared outcomes between patients who did and did not wear a brace during postoperative rehabilitation. In five of these studies, RTR was permitted at the same time for all patients, irrespective of whether they wore a brace or not (7 weeks, ${ }^{63} 12$ weeks ${ }^{64} 6568$ or 16 weeks ${ }^{66}$ postoperative). In one study, patients who did not wear a brace during postoperative rehabilitation commenced running earlier than patients who wore a brace (10 weeks vs 12 weeks). ${ }^{67}$ However, it is important to note that these timelines for RTR were reported as part of the rehabilitation programme, not as a result of a statistical comparison. This suggests the time criteria reported reflected clinical decision-making by the respective researchers. We hypothesise that postoperative bracing does not influence RTR. However, a randomised controlled trial is necessary to test this hypothesis.

\section{When is it safe to RTR?}

Unsurprisingly, we found that the time criterion for RTR was different depending whether a protective or contemporary rehabilitation protocol was employed. With contemporary rehabilitation, patients were permitted to commence running earlier than in protective programmes (where there was a period of postoperative immobilisation). This suggests RTR is-at least to some degree-tied to expectations of underlying physical capacity to meet the demands of running. Few studies used multiple criteria in addition to the time criterion for RTR, suggesting that there is no standard assessment for this key step in the return to sport continuum.

A missing piece of information is whether RTR at a median 12 weeks postoperative is safe. It is unclear how many patients had exacerbations of symptoms, or sustained new knee injuries. This information is important to guide clinical expectations and adequate loading for the individual. To answer the question 'Is RTR at a median 12 postoperative weeks safe?', research designs that incorporate physical activity/rehabilitation exposure, objective assessments (clinical, strength and performance based) and injury (either new acute injuries or gradual-onset injuries) registration are needed.

\section{Methodological considerations}

In line with scoping review methodology, we did not impose date limits on our search or article inclusion. Therefore, we have included articles where surgical and rehabilitation approaches may be different from contemporary clinical practice. We felt it was important to cover the breadth of clinical practice, including how practice has changed over time. We accounted for this in our descriptive synthesis by differentiating between the surgical techniques (open surgery vs arthroscopic surgery) and the rehabilitation protocols (protective vs contemporary rehabilitation protocols).

Differences in how studies defined RTR could influence our results. Not all authors used the same terms to describe the running activity (eg, running, jogging, slow running, and so on). The use of the term 'running' may have different meanings in different articles, and a conceptual definition of a specific term to describe the resumption of running may be important for future research. We propose the term return-to-running activities to describe the phase of RTR at a slow speed $(8-10 \mathrm{~km} /$ hour), but it may be relevant to establish consensus on the definition of the term 'running'. Since authors report general criteria that governed rehabilitation progression, our analysis of the time-based criteria reflects a general time frame, instead of the precise time each patient was permitted to RTR in each study.

One study reported a rehabilitation protocol that included using restricted body weight progression to running. ${ }^{69} \mathrm{New}$ technologies (eg, anti gravity treadmill) may provide the clinician with alternative ways to progressively increase loading during RTR. These technologies might have merit in facilitating adequate loading that enables the patient to commence running earlier. However, it is unclear whether these approaches translate to earlier progression through rehabilitation, and this might be an avenue for future research.

\section{Clinical implications}

RTR decision-making should be individualised for each patient. No universal timeline to RTR exists. For many patients, it might be reasonable to expect readiness to RTR around the 8 th-16th postoperative weeks, provided there is adequate loading: pain $<2$ on visual analogue scale, 95\% knee flexion RoM, full 
knee extension RoM and no effusion. The clinician may choose to use a battery of tests for individualised clinical decision-making regarding RTR including (1) strength tests, (2) qualitative performance-based assessments, with focus on dynamic knee control, and (3) quantitative performance-based assessments such as hop tests. However, it is unclear whether these time-based and assessment-based criteria relate to safe RTR.

\section{CONCLUSION}

In this scoping review including 201 studies from 1981 to 2016, time was the most frequently reported criterion for RTR and fewer than one in five studies reported clinical, strength or performance-based criteria for RTR. It seems reasonable to recommend performance-based criteria combined with timebased criteria to commence running activities following ACL reconstruction.

\section{What are the new findings?}

- Only one in five studies reported clinical, strength or performance-based criteria for return to running (RTR).

- The most cited time-based criterion for RTR was 12 postoperative weeks.

- An approach combining assessment goal-based criteria with time-based criteria is a reasonable approach for RTR after $\mathrm{ACL}$ reconstruction.

Correction notice This article has been updated since it was published Online First. The title has been corrected in order to fix a grammatical error.

Acknowledgements The authors acknowledge and thank Thomas Davergne for his contribution to the help in the searches in the database.

Contributors All authors have contributed to the development of the research questions and study design. AJMR, PE and JPR identified the method of the scoping protocol. AJMR and PE developed and conducted the search strategy and data extraction. All authors developed the first and subsequent drafts of the manuscript. All authors reviewed and approved the manuscript.

Funding The authors have not declared a specific grant for this research from any funding agency in the public, commercial or not-for-profit sectors.

\section{Competing interests None declared.}

\section{Patient consent Not required.}

\section{Provenance and peer review Not commissioned; externally peer reviewed.}

(C) Article author(s) (or their employer(s) unless otherwise stated in the text of the article) 2018. All rights reserved. No commercial use is permitted unless otherwise expressly granted.

\section{REFERENCES}

1 Ardern CL, Taylor NF, Feller JA, et al. Fifty-five per cent return to competitive sport following anterior cruciate ligament reconstruction surgery: an updated systematic review and meta-analysis including aspects of physical functioning and contextual factors. Br J Sports Med 2014;48:1543-52.

2 Ardern CL, Glasgow P, Schneiders A, et al. 2016 Consensus statement on return to sport from the First World Congress in Sports Physical Therapy, Bern. Br I Sports Med 2016;50:853-64.

3 Rambaud AJM, Semay B, Samozino P, et al. Criteria for Return to Sport after Anterior Cruciate Ligament reconstruction with lower reinjury risk (CR'STAL study): protocol for a prospective observational study in France. BMJ Open 2017;7:e015087.

4 Takeda K, Hasegawa T, Kiriyama Y, et al. Kinematic motion of the anterior cruciate ligament deficient knee during functionally high and low demanding tasks. J Biomech 2014;47:2526-30.

5 Myer GD, Paterno MV, Ford KR, et al. Rehabilitation after anterior cruciate ligament reconstruction: criteria-based progression through the return-to-sport phase. J Orthop Sports Phys Ther 2006;36:385-402.

6 Adams D, Logerstedt DS, Hunter-Giordano A, et al. Current concepts for anterior cruciate ligament reconstruction: a criterion-based rehabilitation progression. J Orthop Sports Phys Ther 2012;42:601-14.

7 Grindem H, Granan LP, Risberg MA, et al. How does a combined preoperative and postoperative rehabilitation programme influence the outcome of ACL reconstruction
2 years after surgery? A comparison between patients in the Delaware-Oslo ACL Cohort and the Norwegian National Knee Ligament Registry. Br I Sports Med 2015;49:385-9.

8 Kyritsis P, Bahr R, Landreau P, et al. Likelihood of ACL graft rupture: not meeting six clinical discharge criteria before return to sport is associated with a four times greater risk of rupture. Br J Sports Med 2016:50:946-51.

9 Ardern CL, Taylor NF, Feller JA, et al. Psychological responses matter in returning to preinjury level of sport after anterior cruciate ligament reconstruction surgery. Am J Sports Med 2013;41:1549-58.

10 Arksey H, O'Malley L. Scoping studies: towards a methodological framework. Int I Soc Res Methodol 2005;8:19-32.

11 Colquhoun $\mathrm{HL}$, Levac $\mathrm{D}, \mathrm{O}^{\prime}$ Brien KK , et al. Scoping reviews: time for clarity in definition, methods, and reporting. J Clin Epidemiol 2014;67:1291-4.

12 The Joanna Briggs Institute. Joanna Briggs Institute Reviewers' Manual. 2015 edn. Adelaide, South Australia: The Joanna Briggs Institute, 2015.

13 Anand BS, Feller JA, Richmond AK, et al. Return-to-sport outcomes after revision anterior cruciate ligament reconstruction surgery. Am I Sports Med 2016;44:580-4.

14 Grassi A, Zaffagnini S, Marcheggiani Muccioli GM, et al. After revision anterior cruciate ligament reconstruction, who returns to sport? A systematic review and meta-analysis. Br J Sports Med 2015;49:1295-304.

15 Morrison A, Moulton K, Clark M, et al. English-language restriction when conducting systematic review-based metaanalyses: Systematic review of published studies. Ottawa: Canadian Agency for Drugs and Technologies in Health, 2009.

16 Moher D, Pham B, Lawson ML, et al. The inclusion of reports of randomised trials published in languages other than English in systematic reviews. Health Technol Assess 2003;7:1-90.

17 Horváth G, Koroknai G, Ács B, et al. Prevalence of radiographic primary hip and knee osteoarthritis in a representative Central European population. Int Orthop 2011:35:971-5.

18 Driban JB, Eaton CB, Lo GH, et al. Association of knee injuries with accelerated knee osteoarthritis progression: data from the Osteoarthritis Initiative. Arthritis Care Res 2014;66:1673-9.

19 Duchman KR, Lynch TS, Spindler KP. Graft selection in anterior cruciate ligament surgery: who gets what and why? Clin Sports Med 2017;36:25-33.

20 Andernord D, Desai N, Björnsson $\mathrm{H}$, et al. Patient predictors of early revision surgery after anterior cruciate ligament reconstruction: a cohort study of 16,930 patients with 2-year follow-up. Am J Sports Med 2015;43:121-7.

21 Kaeding CC, Aros B, Pedroza A, et al. Allograft versus autograft anterior cruciate ligament reconstruction: predictors of failure from a moon prospective longitudinal cohort. Sports Health 2011;3:73-81.

22 Murray A, Daines L, Archibald D, et al. The relationship and effects of golf on physical and mental health: a scoping review protocol. Br I Sports Med 2016;50:647-50.

23 De Carlo MS, McDivitt R. Rehabilitation of patients following autogenic bone-patellar tendon-bone acl reconstruction: a 20-year perspective. N Am I Sports Phys Ther 2006;1:108-23.

24 Paulos L, Noyes FR, Grood E, et al. Knee rehabilitation after anterior cruciate ligament reconstruction and repair. Am J Sports Med 1981;9:140-9.

25 Shelbourne KD, Nitz P. Accelerated rehabilitation after anterior cruciate ligament reconstruction. Am J Sports Med 1990;18:292-9.

26 Shelbourne KD, Klootwyk TE, Decarlo MS. Update on accelerated rehabilitation after anterior cruciate ligament reconstruction. J Orthop Sports Phys Ther 1992;15:303-8.

27 Decarlo MS, Shelbourne KD, McCarroll JR, et al. Traditional versus accelerated rehabilitation following ACL reconstruction: a one-year follow-up. J Orthop Sports Phys Ther 1992;15:309-16.

28 Yabroudi MA, Irrgang JJ. Rehabilitation and return to play after anatomic anterior cruciate ligament reconstruction. Clin Sports Med 2013;32:165-75.

29 Sauter AJ, van Haeff MJ, van der Lubbe N, et al. Anterior cruciate ligament reconstruction with alternative tibial tunnel: early results after accelerated weightbearing. Knee Surg Sports Traumato/ Arthrosc 1998;6:220-3.

30 Muneta T, Sekiya I, Ogiuchi T, et al. Effects of aggressive early rehabilitation on the outcome of anterior cruciate ligament reconstruction with multi-strand semitendinosus tendon. Int Orthop 1998;22:352-6.

31 Claes S, Verdonk P, Forsyth R, et al. The "ligamentization" process in anterior cruciate ligament reconstruction: what happens to the human graft? A systematic review of the literature. Am J Sports Med 2011;39:2476-83.

32 Scheffler SU, Unterhauser FN, Weiler A. Graft remodeling and ligamentization after cruciate ligament reconstruction. Knee Surg Sports Traumatol Arthrosc 2008;16:834-42.

33 Bizzini M, Gorelick M, Munzinger U, et al. Joint laxity and isokinetic thigh muscle strength characteristics after anterior cruciate ligament reconstruction: bone patellar tendon bone versus quadrupled hamstring autografts. Clin I Sport Med 2006;16:4-9.

34 Joreitz R, Lynch A, Rabuck S, et al. Patient-specific and surgery-specific factors that affect return to sport after ACL reconstruction. Int I Sports Phys Ther 2016;11:264-78.

35 Lepley LK, Wojtys EM, Palmieri-Smith RM. Combination of eccentric exercise and neuromuscular electrical stimulation to improve quadriceps function post-ACL reconstruction. Knee 2015;22:270-7. 
36 Grindem H, Risberg MA, Eitzen I. Two factors that may underpin outstanding outcomes after ACL rehabilitation. Br J Sports Med 2015;49:1425.

37 van Melick N, van Cingel RE, Brooijmans F, et al. Evidence-based clinical practice update: practice guidelines for anterior cruciate ligament rehabilitation based on a systematic review and multidisciplinary consensus. Br J Sports Med 2016;50:1506-15.

38 Hackney JM, Wade MG, Larson C, et al. Impairment in people with anterior cruciate ligament reconstruction in adjusting ground reaction force in running. Physiother Theory Pract 2010;26:289-96.

39 Lentz TA, Zeppieri G, Tillman SM, et al. Return to preinjury sports participation following anterior cruciate ligament reconstruction: contributions of demographic, knee impairment, and self-report measures. J Orthop Sports Phys Ther 2012:42:893-901.

40 Lentz TA, Zeppieri G, George SZ, et al. Comparison of physical impairment, functional, and psychosocial measures based on fear of reinjury/lack of confidence and return-tosport status after ACL reconstruction. Am J Sports Med 2015;43:345-53.

41 Waters E. Suggestions from the field for return to sports participation following anterior cruciate ligament reconstruction: basketball. J Orthop Sports Phys Ther 2012:42:326-36.

42 Fitzgerald GK, Piva SR, Irrgang JJ. A modified neuromuscular electrical stimulation protocol for quadriceps strength training following anterior cruciate ligament reconstruction. J Orthop Sports Phys Ther 2003;33:492-501.

43 Manske RC, Prohaska D, Lucas B. Recent advances following anterior cruciate ligament reconstruction: rehabilitation perspectives: critical reviews in rehabilitation medicine. Curr Rev Musculoskelet Med 2012;5:59-71.

44 Sigward SM, Lin P, Pratt K. Knee loading asymmetries during gait and running in early rehabilitation following anterior cruciate ligament reconstruction: a longitudinal study. Clin Biomech 2016:32:249-54.

45 DeMaio M, Mangine RE, Noyes FR, et al. Advanced muscle training after ACL reconstruction: weeks 6 to 52. Orthopedics 1992;15:757-67.

46 Wilk KE, Arrigo C, Andrews JR, et al. Rehabilitation after anterior cruciate ligament reconstruction in the female athlete. J Ath/ Train 1999;34:177-93.

47 Manal TJ, Snyder-Mackler L. Practice guidelines for anterior cruciate ligament rehabilitation: a criterion-based rehabilitation progression. Oper Tech Orthop 1996;6:190-6.

48 Anderson JL, Lamb SE, Barker KL, et al. Changes in muscle torque following anterior cruciate ligament reconstruction: a comparison between hamstrings and patella tendon graft procedures on 45 patients. Acta Orthop Scand 2002;73:546-52.

49 Taylor DC, DeBerardino TM, Nelson BJ, et al. Patellar tendon versus hamstring tendon autografts for anterior cruciate ligament reconstruction: a randomized controlled trial using similar femoral and tibial fixation methods. Am J Sports Med 2009;37:1946-57.

50 Hartigan EH, Axe MJ, Snyder-Mackler L. Time line for noncopers to pass return-tosports criteria after anterior cruciate ligament reconstruction. J Orthop Sports Phys Ther 2010;40:141-54

51 Amano K, Li Q, Ma CB. Functional knee assessment with advanced imaging. Curr Rev Musculoskelet Med 2016:9:123-9.

52 Tourville TW, Jarrell KM, Naud S, et al. Relationship between isokinetic strength and tibiofemoral joint space width changes after anterior cruciate ligament reconstruction. Am J Sports Med 2014;42:302-11.

53 Decker LM, Moraiti C, Stergiou N, et al. New insights into anterior cruciate ligament deficiency and reconstruction through the assessment of knee kinematic variability in terms of nonlinear dynamics. Knee Surg Sports Traumatol Arthrosc 2011;19:1620-33.

54 Czamara A. Moments of muscular strength of knee joint extensors and flexors during physiotherapeutic procedures following anterior cruciate ligament reconstruction in males. Acta Bioeng Biomech 2008;10:37-44.
55 Karasel S, Akpinar B, Gülbahar S, et al. Clinical and functional outcomes and proprioception after a modified accelerated rehabilitation program following anterior cruciate ligament reconstruction with patellar tendon autograft. Acta Orthop Traumatol Turc 2010:44:220-8.

56 Lemiesz $\mathrm{G}$, Lemiesz $\mathrm{E}$, Wołosewicz $\mathrm{M}$, et al. The effectiveness of rehabilitation procedure after the reconstruction of the anterior cruciate ligament according to the Norwegian protocol. Pol Ann Med 2011;18:82-95.

57 Gokeler A, Welling W, Zaffagnini S, et al. Development of a test battery to enhance safe return to sports after anterior cruciate ligament reconstruction. Knee Surg Sports Traumatol Arthrosc 2017;25:192-9.

58 Grindem H, Snyder-Mackler L, Moksnes H, et al. Simple decision rules can reduce reinjury risk by $84 \%$ after $A C L$ reconstruction: the Delaware-Oslo $A C L$ cohort study. $B r$ J Sports Med 2016;50:804-8.

59 Wilk KE, Andrews JR. Current concepts in the treatment of anterior cruciate ligament disruption. J Orthop Sports Phys Ther 1992;15:279-93.

60 Risberg MA, Holm I, Myklebust G, et al. Neuromuscular training versus strength training during first 6 months after anterior cruciate ligament reconstruction: a randomized clinical trial. Phys Ther 2007;87:737-50.

61 Fu FH, L-Y Woo S, Irrgang JJ. Current concepts for rehabilitation following anterior cruciate ligament reconstruction. J Orthop Sports Phys Ther 1992;15:270-8.

62 Wilk KE, Reinold MM, Hooks TR. Recent advances in the rehabilitation of isolated and combined anterior cruciate ligament injuries. Orthop Clin North Am 2003;34:107-37.

63 Feller J, Bartlett J, Chapman S, et al. Use of an extension-assisting brace following anterior cruciate ligament reconstruction. Knee Surg Sports Traumatol Arthrosc 1997:5:6-9

64 Kartus J, Stener S, Köhler K, et al. Is bracing after anterior cruciate ligament reconstruction necessary? A 2-year follow-up of 78 consecutive patients rehabilitated with or without a brace. Knee Surg Sports Traumatol Arthrosc 1997:5:157-61.

65 Brandsson S, Faxén E, Kartus J, et al. Is a knee brace advantageous after anterior cruciate ligament surgery? A prospective, randomised study with a two-year followup. Scand J Med Sci Sports 2001;11:110-4.

66 Henriksson M, Rockborn P, Good L. Range of motion training in brace vs. plaster immobilization after anterior cruciate ligament reconstruction: a prospective randomized comparison with a 2-year follow-up. Scand J Med Sci Sports 2002;12:73-80.

67 Hasan HA. Tegner and Lysholm scores in brace-free rehabilitation. Saudi Med J 2004;25:1962-6

68 Vadalà A, lorio R, De Carli A, et al. The effect of accelerated, brace free, rehabilitation on bone tunnel enlargement after $\mathrm{ACL}$ reconstruction using hamstring tendons: $\mathrm{a} C \mathrm{CT}$ study. Knee Surg Sports Traumatol Arthrosc 2007;15:365-71.

69 Wilk KE, Macrina LC, Cain EL, et al. Recent advances in the rehabilitation of anterior cruciate ligament injuries. J Orthop Sports Phys Ther 2012;42:153-71.

70 De Carlo MS, Sell KE, Shelbourne KD, et al. Current concepts on accelerated ACL rehabilitation. J Sport Rehabil 1994:3:304-18.

71 Seto JL, Brewster CE, Lombardo SJ, et al. Rehabilitation of the knee after anterior cruciate ligament reconstruction. J Orthop Sports Phys Ther 1989;11:8-18.

72 Souissi S, Wong delP, Dellal A, et al. Improving functional performance and muscle power 4-to-6 months after anterior cruciate ligament reconstruction. J Sports Sci Med 2011;10:655-64.

73 Bollen SR. BASK Instructional Lecture 3: rehabilitation after ACL reconstruction. Knee 2001:8:75-7.

74 Gorschewsky 0, Klakow A, Riechert K, et al. Clinical comparison of the Tutoplast allograft and autologous patellar tendon (bone-patellar tendon-bone) for the reconstruction of the anterior cruciate ligament: 2- and 6-year results. Am J Sports Med 2005:33:1202-9. 